\title{
Prevalence and Risk Factors of Pregnancy Associated Malaria in Pregnant Women Attending a General Hospital
}

\author{
Valentine $\mathrm{UN}^{1 *}$, Ndidi $\mathrm{OC}^{2}$, Ndubuisi $\mathrm{NO}^{3}$ and Ugbo $\mathrm{EN}^{4}$ \\ ${ }^{1}$ Department of Microbiology, Spiritan University Nneochi, Abia State, Nigeria \\ ${ }^{2}$ Department of Microbiology, Veritas University Abuja, Nigeria \\ ${ }^{3}$ Department of Microbiology, Abia State University/Uturu, Nigeria \\ ${ }^{4}$ Department of Applied Microbiology, Ebonyi State University, Nigeria
}

\section{Research Article \\ Volume 5 Issue 4}

Received Date: September 24, 2020

Published Date: November 20, 2020

DOI: $10.23880 /$ oajmb-16000175

*Corresponding author: Unegbu Valentine Nnachetam, Department of Microbiology, Spiritan University Nneochi, Abia State, Nigeria, Tel: 08035402207; Email: donval4u@yahoo.com

\section{Abstract}

Malaria in pregnancy poses a serious public health issues especially in developing countries. This study was to determine the Prevalence and risk factors of Malaria among Pregnant women attending antenatal clinic in Aboh Mbaise General Hospital. A total of 284 pregnant women participated in this study. A structured interviewer-administered questionnaire was used to obtain some socio-demographic characteristics of patients. Thick and thin films were made from blood collected from each pregnant woman. The films were stained using the gold standard staining technique, Giemsa staining for detecting malaria parasites in blood. Data generated was analyzed using SPSS 23.0 statistical package. A p-value $<0.05$ was considered significant. The prevalence of malaria parasite among the pregnant women in Aboh Mbaise was $70.8 \%$. Plasmodium falciparum was the only species of malaria parasite found in this study. The age group $15-25$ years, illiterates, unemployed and single patients had the highest prevalence of malaria positive (MP) patients of 81(28.5\%), 80(28.2\%), 140(49.3\%) and 53(18.7\%) respectively. Primigravidae had the highest frequency of MP patients of $109(38.4 \%)$ followed by multigravidae $92(32.4 \%)$ but there was no statistical significance ( $p=1.003)$. Malaria prevalence followed the trend of 1 st trimester $80(28.2 \%)$ to $2 \mathrm{nd}$ trimester $66(23.2 \%)$ to 3rd trimester $45(15.8 \%)$ in that order but the differences were not statistically significant $(p=1.002)$. The lowest frequency occurred in the month of February $9(3.2 \%)$ while the highest frequency occurred in the month of July $62(21.8 \%)$ but the difference was not statistically significant $(\mathrm{p}=0.803)$. Patients who had bushes around their compound, and who didn't use mosquito bed net (MBN) and insecticide treated nets (ITNs); those patients surrounded by mosquito breeding sites and who didn't use Intermittent Preventive Treatment (IPT) were significantly associated with malaria parasite infection $(p<0.05)$. The prevalence of malaria parasite among the pregnant women in was $70.8 \%$. Lack of education and employment; and risk factors like non-compliance to use of ITNs/MBN; and non-usage of IPT during pregnancy, presence of mosquito breeding sites and bushes around the premises of the study participants were risk factors contributing to prevalence of malaria among pregnant women in Aboh Mbaise General Hospital in Imo State.

Keywords: Malaria; Multigravidae; Plasmodium falciparum; Primigravidae; Pregnant Women; Prevalence; Risk Factors 


\section{Open Access Journal of Microbiology \& Biotechnology}

Abbreviations: GDP: Gross Domestic Product; PAM: Pregnancy-associated Malaria; LBW: Low Birth Weight; WHO: World Health Organization; LGA: Local Government Area; ANC: Antenatal Care; MBN: Mosquito Bed Net; ITNs: Insecticide Treated Bed Nets; IPT: Intermittent Preventive Treatment; IPTp: Intermittent Preventive Therapy in Pregnancy; IPTp-SP: Intermittent Preventive Treatment with Sulfadoxine-Pyrimethamine.

\section{Introduction}

Malaria, an acute febrile illness caused by protozoa of the genus Plasmodium, continues to constitute a major source of morbidity and mortality globally [1]. Five species of the genus Plasmodium are pathogenic to human, namely, P. falciparum, P. vivax, P. malariae, P. ovale and the recently included $P$. knowlesi. $P$. falciparum causes the most virulent cases of human malaria [2]; it is of major importance in subSaharan Africa and serves as the species of interest in this research.

Presently, malaria poses enormous burden to societies, especially to the developing countries. It is especially regrettable in sub-Saharan Africa. In Nigeria, over $12.0 \%$ of the gross domestic product (GDP) is expended on malaria annually [3]. This enormous cost of managing malaria further contributes to the negating of developmental efforts in Nigeria [3].

The recent World Malaria Report, which indicated that Nigeria accounts for a quarter of all malaria cases in the 45 malaria-endemic countries in Africa, clearly showed the challenge of malaria in Nigeria [4]. This may be due to the large population, approximately 140 million people living in areas of high malaria transmission [4]. In Nigeria, 11\% of maternal deaths are attributed to malaria [5]. To further buttress the worrisome malaria picture, many researchers have reported high prevalence rates of malaria in pregnancy in different parts of Nigeria, ranging from $19.7 \%$ to $72.0 \%$ $[6,7]$. Thus, pregnant women, who are known to be one of the groups at high risk of the effects of malaria infection, need special protective measures to ensure their survival and improve birth outcome.

Pregnancy-associated malaria (PAM)-malaria infection during pregnancy is a major public health concern constituting a serious risk to the pregnant woman, her foetus and the newborn [8]. This is as infection by malaria parasites especially P. falciparum further subjects the pregnant woman to stresses in addition to that arising from pregnancy. This combination of stresses which on one hand is directed at sustaining the foetus and on the other at eliminating parasite may overwhelm the pregnant woman leading to her death, or abortion of the foetus, still birth and low birth weight (LBW) neonates [2].

Transfer of Plasmodium from maternal circulation through the placenta into foetal circulation is yet to be fully established, but the possibility of such scenario is greatly favored by research findings $[9,10]$.

The increased risk of malarial infection among pregnant women could be related to illiteracy, low educational status, unemployment, low income and gravidity of the pregnant women $[11,12]$. Young maternal age has been identified as an independent risk factor for malarial infection [13]. Malaria also exerts a huge social and economic burden on families, communities, and the country at large, causing an annual loss of about 132 billion naira in payments for treatment and prevention as well as hours not worked [14].

The efforts to control malaria by the Government and other agencies like the Roll Back Malaria programme, World Health Organization (WHO), United Nations International Children Emergency Fund (UNICEF), and many other nongovernmental agencies might not be effective. While there are reports of up to $50 \%$ reduction in malaria episodes and deaths in some African countries between 2000 and 2006 [15], reports from Nigeria has not shown significant reduction, especially with regards to malaria in pregnancy. The reasons adduced for the change in malaria prevalence in other countries are good surveillance and high intervention coverage [15].

Considering the wide variation in reports of the prevalence of malaria in pregnancy in Nigeria and the failure of government to reduce death associated with PAM significantly, it became imperative for a closer assessment of the prevalence and risk factors of malaria among pregnant women attending antenatal clinic in Aboh Mbaise General Hospital of Imo State.

\section{Materials and Methods}

\section{Study Area}

The study was carried out at Aboh Mbaise General Hospital located at Aboh Mbaise Local Government Area (LGA) of Imo State. It is usually attended by very low and moderate socio-economic groups and therefore very affordable and accessible to most dwellers of Ahiazu Mbaise LGA, Ezinihitte Mbaise LGA and Aboh Mbaise LGA.

\section{Sample Size}

The population size consisted of 284 pregnant women and 16 age-matched healthy non-parasitized pregnant women who were monitored as control within the ages 


\section{Open Access Journal of Microbiology \& Biotechnology}

( $\geq 15$ years) and who attended the antenatal clinics of Aboh Mbaise General Hospital.

\section{Study Period}

This research was carried out between $1^{\text {st }}$ February 2019 and $31^{\text {st }}$ July 2019.

\section{Study Subjects/Selection}

\section{Inclusion Criteria}

Women who met the following inclusion criteria were recruited in the study;

* Pregnant women ( $\geq 15$ years) attending Antenatal Clinic at Aboh Mbaise General Hospital, Imo State, Nigeria.

* Pregnant women who gave written informed consent and agreed to be included in the study.

* Pregnant women with no history of hereditary diseases and/or no known severe disease at the time of conceiving and/or at first Antenatal care (ANC) attendance.

\section{Exclusion Criteria}

The following were excluded from participating as subjects in the study.

* Pregnant women below 15 years attending Antenatal Clinic at Aboh Mbaise General Hospital, Imo State, Nigeria.

* Pregnant women who did not offer an informed consent to be included in the study.

* Clinically suspected or identified cases of HIV and hepatitis B infection, because these conditions may affect the hemoglobin concentrations of affected persons.

\section{Study Instrument (i.e Questionnaire)}

A semi- structured interviewer-administered questionnaire was administered to all consenting participants in order to obtain information on their sociodemographic (i.e., date of birth, occupation, marital status, educational status, location), reproductive history including gravidity, antimalarial drug use, and use of antimalarial prevention measures).

\section{Informed Consent}

Written informed consent was obtained from all the study participants (subjects and controls).

\section{Blood Samples Collection}

Two (2) millimeters blood was collected from pregnant women attending antenatal clinic. Using a soft tubing tourniquet fastened on to the upper arm of the subject to dilate a suitable vein, a venal puncture was made on the surface of the arm cleaned with a methylated spirit. Both thick and thin films were made for each subject as described [16].

\section{Parasitological Examination}

The thick and thin films were stained using the gold standard technique- Giemsa staining. While thick films were used to detect malaria parasites in the blood samples (ring forms trophozoites and gametocytes), parasite species and morphology were determined by microscopic examination of thin films.

The slides were immersed for 30 minutes in a staining jar containing Giemsa stain freshly diluted with 20 volumes of water buffered to $\mathrm{pH}$ 7.2.The slides were washed in buffered water $\mathrm{pH} 7.2$ for 3 minutes. Thereafter, they were made to stand upright to dry in a draining rack for eventual examination of the slides under microscope "Cx 22 Olympus Binocular Microscope", using oil immersion at X100 magnifications to observe Plasmodium parasites (16). The stained slides were observed by two (2) certified Medical Laboratory Scientists and observations recorded.

A blood smear was considered negative when the observation of 100 high power fields did not reveal malaria parasites.

\section{Malaria Parasite Density Count}

The absolute parasite density was calculated using the formula: [No. of parasites counted x total leukocyte count] /No. of leucocytes counted [16]. Blinded readings for validation of the presence or absence of parasites and of the estimated parasite density were done by Certified Medical Laboratory Scientist.

\section{Quality Control}

For quality control, blind rechecking of all slides was carried out by an independent Certified Medical Laboratory Scientist who also served as a tie breaker.

\section{Ethical Clearance}

Approval for the study was obtained from the Hospital Ethical Committee. The participants were provided with consent form and fact sheet concerning information about 


\section{Open Access Journal of Microbiology \& Biotechnology}

the study.

\section{Data Analysis}

The generated data were analyzed using statistical package for social sciences (SPSS) version 23, and Chi-square $(\chi 2)$ test was used to determine the relationship between variables. A $\mathrm{P}$ value of $\mathrm{P} \leq 0.05$ was taken as significant level.

\section{Results}

Table 1 shows the Association between Socio- demographic characteristics of study population and malaria infection. Exactly 201(70.8\%) pregnant women were positive for malaria parasite and only one species of malaria parasite (Plasmodium falciparum) was discovered in this study. There was no statistical significance between prevalence of malaria parasite in relation to age $(p>0.05)$. There was also no significant association between prevalence of malaria parasite in relation to marital status ( $p>0.05)$. Occupation and employment status were significantly associated with malaria $(\mathrm{p}<0.05)$. Other parameters are found in the Table 1.

\begin{tabular}{|c|c|c|c|c|c|}
\hline Characteristics & No. of patients (\%) & No. of MP patients (\%) & $\chi 2$ & df & P-Value \\
\hline \multicolumn{6}{|l|}{ Age } \\
\hline $15-25$ & $109(38.4)$ & $81(28.5)$ & 6.431 & 1 & 1.001 \\
\hline $26-35$ & $95(33.5)$ & $68(23.9)$ & & & \\
\hline$\geq 36$ & $80(28.2)$ & $52(18.3)$ & & & \\
\hline Total & 284 & $201(70.8)$ & & & \\
\hline \multicolumn{6}{|l|}{ Marital Status } \\
\hline Single & $100(35.2)$ & $53(18.7)$ & 7.154 & 2 & 3.002 \\
\hline Married & $59(20.8)$ & $51(18.0)$ & & & \\
\hline Cohabiting & $39(13.7)$ & $31(17.9)$ & & & \\
\hline Divorce & $15(5.3)$ & $11(3.9)$ & & & \\
\hline Separated & $61(21.5)$ & $50(17.6)$ & & & \\
\hline Widow & $10(3.5)$ & $5(1.8)$ & & & \\
\hline Total & 284 & $201(70.8)$ & & & \\
\hline \multicolumn{6}{|l|}{ Educational Status } \\
\hline None & $100(35.2)$ & $80(28.2)$ & 17.733 & 4 & 0.002 \\
\hline Primary & $92(32.4)$ & $76(26.8)$ & & & \\
\hline Secondary & $39(13.7)$ & $16(5.6)$ & & & \\
\hline Tertiary institution & $43(15.1)$ & $29(10.2)$ & & & \\
\hline Total & 284 & $201(70.8)$ & & & \\
\hline \multicolumn{6}{|l|}{ Occupation } \\
\hline Employed & $134(41.2)$ & $61(21.5)$ & 16.691 & 3 & 0.006 \\
\hline Unemployed & $150(52.8)$ & $140(49.3)$ & & & \\
\hline Total & 284 & $201(70.8)$ & & & \\
\hline \multicolumn{6}{|l|}{ Location } \\
\hline Aboh Mbaise & $139(48.9)$ & $114(40.1)$ & 9.667 & 1 & 4.664 \\
\hline Ezinihitte Mbaise & $84(29.6)$ & $51(18.0)$ & & & \\
\hline Ahiazu Mbaise & $61(21.7)$ & $36(12.7)$ & & & \\
\hline Total & 284 & $201(70.8)$ & & & \\
\hline
\end{tabular}

Table 1: Association between Socio-demographic characteristics of study population and malaria.

Note: MP = Malaria parasite. 


\section{Open Access Journal of Microbiology \& Biotechnology}

Table 2 highlighted the prevalence of malaria infection in relation to gravidity. Gravidity was not statistically significant $(p>0.05)$ where primigravid women had the highest prevalence of malaria infection 109 (38.4\%) followed by multigravid women $92(32.4 \%)$.

\begin{tabular}{|c|c|c|c|c|c|}
\hline Gravidae & No. of patients & No. of MP positive Patients (\%) & $\chi \mathbf{2}$ & df & P-Value \\
\hline Primigravidae & 130 & $109(38.4)$ & 7.028 & 4 & 1.003 \\
\hline Multigravidae & 154 & $92(32.4)$ & & & \\
\hline Total & $\mathbf{2 8 4}$ & $\mathbf{2 0 1 ( 7 0 . 8 )}$ & & & \\
\hline
\end{tabular}

Table 2: Distribution of Malaria parasite (MP) patients according to Gravidae.

Note: MP = Malaria parasite.

Table 3 showed the prevalence of malaria infection in various trimesters. Malaria prevalence followed the trend of 1 st trimester $80(28.2 \%)$ to 2 nd trimester $66(23.2 \%)$ to 3rd trimester $45(15.8 \%)$ in that order of occurrence. But the difference was not statistically significant $(p>0.05)$.

\begin{tabular}{|c|c|c|c|c|c|}
\hline Trimester & No. of patients & No. of MP positive Patients & $\chi \mathbf{2}$ & df & P-Value \\
\hline $1^{\text {st }}$ trimester & $119(41.9)$ & $80(28.2)$ & 9.341 & 3 & 1.002 \\
\hline $2^{\text {nd }}$ trimester & $74(26.1)$ & $66(23.2)$ & & & \\
\hline $3^{\text {rd }}$ trimester & $91(32.0)$ & $45(15.8)$ & & & \\
\hline Total & 284 & $201(70.8)$ & & & \\
\hline
\end{tabular}

Table 3: Distribution of Malaria Parasite (MP) patients according to Trimester.

Note: MP = Malaria parasite.

Table 4 shows MP patients attending antenatal care according to Month. The was an increase in the frequency of MP positive patients from February to July as the lowest frequency occurred in the month of February 9(3.2\%) while the highest frequency occurred in the month of July $62(21.8 \%)$ but the difference was not statistically significant $(\mathrm{p}=1.002)$.

\begin{tabular}{|c|c|c|c|c|c|}
\hline Month & No. of patients (\%) & No. of MP positive Patients (\%) & $\chi 2$ & df & P- Value \\
\hline February & 20 & $9(3.2)$ & 8.153 & 4 & 0.803 \\
\hline March & 24 & $10(3.5)$ & & & \\
\hline April & 33 & $17(6.0)$ & & & \\
\hline May & 62 & $50(17.6)$ & & & \\
\hline June & 68 & $53(18.7)$ & & & \\
\hline July & 77 & $62(21.8)$ & & & \\
\hline Total & 284 & $201(70.8)$ & & & \\
\hline
\end{tabular}

Table 4: Distribution of MP patients according to Months.

Note: MP = Malaria parasite.

Table 5 highlighted the risk factors to malaria infection in pregnant women. Patients who had bushes around their compound, and who didn't use mosquito bed net (MBN) and insecticide treated net, those patients whose premises were surrounded by mosquito breeding sites and who didn't use IPT were significantly associated with malaria parasite infection $(\mathrm{p}<0.05)$. 


\section{Open Access Journal of Microbiology \& Biotechnology}

\begin{tabular}{|c|c|c|c|c|c|}
\hline Characteristics & No. of patients & Positive No (\%) & $\chi \mathbf{2}$ & df & P- Value \\
\hline State of the compound & 284 & & 22.073 & 6 & 0.007 \\
\hline (Bushes around compound) & & & & & \\
\hline Yes & 182 & 158 & & & \\
\hline No & 82 & 43 & & & \\
\hline Possession of mosquito bed net & 284 & & & & \\
\hline Yes & 82 & 54.391 & 5 & 0.006 \\
\hline No & 202 & 147 & & & \\
\hline Presence of breeding sites & 284 & & 20.044 & 4 & 0.005 \\
\hline (i.e open gutters, stagnant water) & & & & \\
\hline Yes & 218 & 166 & & \\
\hline No & 66 & 35 & 22.211 & 2 & 0.000 \\
\hline Sleeping under ITN & 284 & & & \\
\hline Yes & 72 & 37 & 19.004 & 3 & 0.003 \\
\hline Use of IPT & 212 & 164 & & & \\
\hline Yes & 248 & & & \\
\hline No & 99 & 51 & 150 & & \\
\hline
\end{tabular}

Table 5: Risk factors to malaria in pregnancy.

Note: ITN = Insecticide Treated Net; ITP = Intermittent Preventive Treatment (i.e, sulfadoxine-pyrimethamine (IPTp-SP).

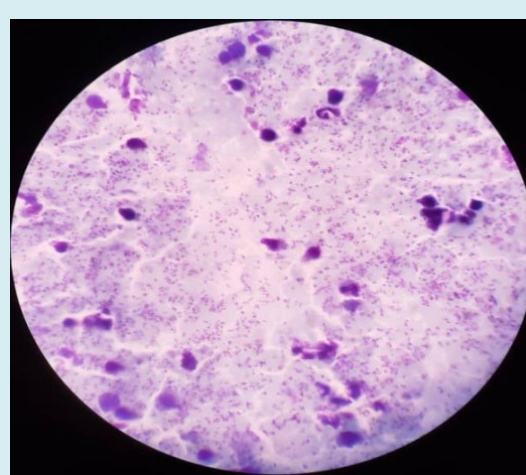

Figure 1: Thick film using Giemsa stain under the microscope.

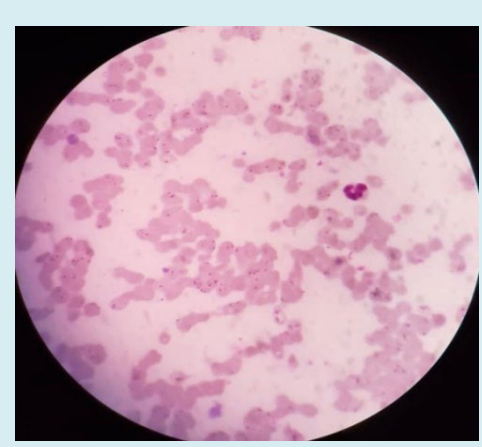

Figure 2: Thin film using Giemsa stain under the microscope.

\section{Discussion}

In this study, the prevalence of malaria parasite among pregnant women was 201(70.8\%), with only one species of malaria parasite (Plasmodium falciparum) present. This is similar to the works of Umaru and Uyaiabasi, in Kaduna State [13]. Pregnant women between the ages of 15 - 25 years of age were found to be at risk of malaria infection but this was not statistically significant with other age groups $(p>0.05)$. This finding is consistent with earlier reports $[17,18]$ where age group of $<24$ year was reported to be at a high risk compared to older pregnant women. Adults living in malaria endemic regions have a protective immunity against malarial infection. This shows that risk of malaria decreases with increase in age. Clinically, the presentation of malaria infection varies by age and the status of the pregnancy with older age providing some protection against malaria [19]. The immunity accumulates with repeated pregnancies, so long as there is exposure to malaria infection. It has also been described as advanced depressed immunity due to delayed antibody expression or lack of awareness on the necessary preventive measures in pregnancy, which could be responsible for the increased malaria parasitemia in the primigravidae [19].

The high rate of malaria among single ladies could also be attributed to their young age as most of the single ladies fell within the age range of 15 to 25 years. This was also not 


\section{Open Access Journal of Microbiology \& Biotechnology}

statistically significant $(\mathrm{p}<0.05)$. This also shows that risk of malaria decreases with increase in age. Similar results were found in the work of Chimere et al. [20].

Pregnant women with low educational background were significantly associated $(\mathrm{p}<0.05)$ with malaria infection. It was observed that pregnant women who had no formal educational and those with primary school leaving certificates had the highest risk of acquiring malaria compared to those with secondary or tertiary school certificate. The high infection rate among pregnant women with no formal education and primary school in this study was similar to that of Gajida et al. [21], who reported 54.7\% of the pregnant women attended Quranic schools only. This might be due to the fact that pregnant women with tertiary school certificate are more likely to reside in areas that do not support mosquito breeding and areas with good sanitary hygiene better sanitary conditions [21]. However, this contradicts the findings of Iriemenam et al. [22], in Lagos, Southwest, Nigeria, who found no significant association between the educational status of the pregnant women and malaria.

The high infection rate among unemployed pregnant women in this current study was similar to that of Fana et al. [23], who reported $15 \%$ of the respondents were physical working class, $17 \%$ craft women, $77 \%$ full house wives, $32 \%$ traders, health workers $0.2 \%, 0.3 \%$ teachers and students $0.3 \%$. Unemployment in this study was significantly associated $(\mathrm{p}<0.05)$ with the high prevalence of malaria infection in pregnancy, which corroborates the findings of Fana et al. [23]. The employment status of an individual is one of the contributing factors of the person's financial background. A pregnant woman with well- established economic status is likely to have better quality of life such as proper health care services, ability to buy insecticide spray, mosquito coil and insecticide treated bed nets (ITNs).

Aboh Mbaise recorded the highest number of prevalence. This is because Aboh Mbaise is where the hospital is located and had the highest number of patients that attend the clinic for ante natal care during the study period. More so, visit to Aboh Mbaise shows dilapidated roads with no drainage facilities. This results to the presence of big stagnant and running ponds around the area, which is a good breeding ground for mosquito. This is similar to the work of Gajida, et al. [21], who reported a high incidence in Sauna quarters in Kano State.

Primigravidae have also been found to be at greatest risk of malaria in pregnancy due to the absence of specific immunity to placental malaria, which is acquired from exposure to malaria infection during pregnancy. The findings in this study is similar to that of Oyefabi et al. [12], in Makurdi, Benue State, Nigeria, who reported pregnant women in primigravida and gravida 2 had the highest risk of malarial infection followed by the multigravidas. In line with this study, similar reports show primigravidae had greater risk of having malaria than the multigravida [24,25]. This correlate with a study in Katsina State, who reported higher risk of malaria infection among primigravidae than multigravida which may be due to the fact that pregnant women at primigravidae are mostly stressed up as a result of the physiological changes that may occur during their first pregnancy which lowers their immunity [26]. This could also be due to the fact that the multigravidas are likely to be much more familiar with preventive and control measures against malaria due to information they acquired during the previous antenatal visits as compared to primigravidae who were attending for the first time.

In this study, it was observed that pregnant women in their first trimester had the highest risk of malarial infection. This is in line with the study of Amuta and colleagues who reported higher risk of malarial infection among pregnant women in their first trimester as compared to those in their second and third trimester [12]. This might be due to the fact that the pregnant women started taking intermittent preventive treatment (IPT) during their second and third trimester which helps to reduce the risk of malarial infection [27]. On the contrary, a study conducted in Lagos, Nigeria, reported higher risk of malaria during third trimester of pregnancy [28]. It has been reported that pregnant women in their second trimester were found to be the highest risk group of infection followed by third trimester while those in first trimester were the lowest risk group [24]. Previous study reported malarial infections vary at different trimesters of pregnancy, which might be as a result of some factors such as exposure to mosquito bite which might be due to the environment, use of preventive measures and immunogenetic constitution of the pregnant women [12].

From the results, the month of May, June and July recorded the highest prevalence in that order. This could be attributed to the advancement of rainy season usually starts in May and progresses to June and July. As heavy shower usually occurs during this period, everywhere is usually green due to various vegetation which allows breeding ground and hiding place for the mosquito. It is therefore during this period that high rate of man-mosquito contact is obtained, which is one of the determinants for malarial transmission [12]. These findings agree with work of [27].

Several studies have also justified that having houses surrounded with vegetation serves as hiding ground for mosquitos. The study found that pregnant women living in houses surrounded with bushes have an increased odd of having malaria. The findings are consistent with the findings 


\section{Open Access Journal of Microbiology \& Biotechnology}

of a study on individual and housing factors influencing the incidence of malaria in Ethiopia and in another study in Bomaka and Molyko in Cameroon [27,28].

The presence of breeding sites like open drainages and stagnant water etc, exerted a profound effect on malaria prevalence among pregnant women in this study. The odds of having malaria were found to be high in pregnant women who have breeding closed to their residence compared to those without breeding sites. The findings were consistent with other studies in Cameroon, Ethiopia and Uganda. The studies found an increased risk of acquiring malaria among people living around mosquito breeding sites [27-29].

In this study, we observed that pregnant women who are not on prophylactic antimalarial medication were significantly more likely to have malaria compared to those that do $(\mathrm{p}<0.05)$. Our finding is consistent with a previous report [17] which suggesting the continuation of IPTp to reduce pregnancy-associated malaria morbidity. An evidenced based way of controlling malaria in pregnancy is the administration of intermittent preventive treatment with sulfadoxine-pyrimethamine during pregnancy (IPTp-SP). WHO recommends IPTp with sulfadoxine-pyrimethamine (IPTp-SP) in all areas with moderate to high malaria transmission in Africa [29]. This consists of a full therapeutic course of antimalarial medicine given to pregnant women at routine prenatal visits, regardless of whether they are infected with malaria or not. Intermittent preventive treatment reduces incidences of maternal malaria episodes, maternal and foetal anaemia, placental parasitaemia, low birth weight and neonatal mortality. Despite the fact that intermittent preventive therapy in pregnancy (IPTp) with sulfadoxinepyrimethamine (IPTp-SP) is a clinically-proven method to prevent the adverse outcomes of malaria in pregnancy for the mother, her foetus, and the neonates, progress towards implementation of universal coverage remains suboptimal with widespread regional and socioeconomic disparities in the utilization of this highly cost-effective intervention [17].

This study has also found that use of mosquito bed net (MBN) and insecticide- treated mosquito net (ITN) has great influence in preventing malaria. Malaria prevalence was significantly higher among women who do not use both MBN and ITN $(\mathrm{p}<0.05)$. This confirms the report of World Health Organization [30], on the use of both nets as a means to reduce the lethal impact of malaria. Our finding is consistent with previous reports in Yaounde, Cameroon [17], which indicated the important role that long-lasting insecticide treated nets can play in the prevention of malaria. Unfortunately, few people in high-risk areas have access and use MBN and ITNs. This is a public health challenge that requires prompt attention. Previous report however indicates that the challenge does not necessarily mean lack of availability of both nets but low utilization in Aboh Mbaise Imo State Nigeria [7].

\section{Conclusion}

The prevalence of malaria parasite among pregnant women in Aboh Mbaise LGA of Imo State was 201(70.8\%). Plasmodium falciparum was the only species of malaria parasite found in this study. The age group 15-25 years, illiterates, unemployed and single patients had the highest prevalence of malaria positive (MP) patients of $81(28.5 \%)$, $80(28.2 \%), 140(49.3 \%)$ and 53(18.7\%) respectively. Lack of education and employment; and risk factors such as non-compliance to use of insecticide-treated mosquito nets (ITNs)/mosquito bed net (MBN); and non-usage of Intermittent preventive drugs (ITPs) during pregnancy, presence of mosquito breeding sites and bushes around the premises of the study participants were risk factors contributing to prevalence of malaria among pregnant women in Aboh Mbaise General Hospital in Imo State.

\section{Recommendations}

Pregnant women in Aboh Mbaise should be educated and informed on preventive measures against malaria parasite.

\section{References}

1. WHO (2016) Fact sheet: World Malaria Report 2015. World Health Organization.

2. Schantz Dunn J, Nour NM (2009) Malaria and pregnancy: A global health perspective. Rev Obstet Gynecol 2(3): 186-192.

3. Jimoh A, Sofola O, Petu A, Okorosoboet T (2007) Quantifying the economic burden of malaria in Nigeria using the willingness to pay approach. Cost Eff Resour Alloc 5: 6.

4. WHO (2008) World Malaria Report 2008. World Health Organization, Switzerland, pp: 190.

5. (2000) Malaria situation analysis document. Federal Ministry of Health, Nigeria, pp: 14

6. Okwa 00 (2003) The status of malaria among pregnant women: a study in Lagos, Nigeria. Afr J Reprod Health 7(3): 77-83.

7. Kagu MB, Kawuwa MB, Gadzama GB (2007) Anaemia in pregnancy: a cross-sectional study of pregnant women in a Sahelian tertiary hospital in Northeastern Nigeria. J Obstet Gynecol 27(7): 676- 679.

8. WHO (2014) WHO policy brief for the implementation 


\section{Open Access Journal of Microbiology \& Biotechnology}

of intermittent preventive of malaria in pregnancy using sulfadoxin-pyrimethamine (IPTp-SP). WHO Global Malaria Programme.

9. Mwangoka GW, Kimera SI, Mboera LE (2008) Congenital Plasmodium falciparum infection in neonates in Muheza District, Tanzania. Malar J 7: 117.

10. Lo YM, Lau TK, Chan LY, Leung TN, Chang AM (2000) Quantitative analysis of the bidirectional fetomaternal transfer of nucleated cells and plasma DNA. Clin Chem 46(9): 1301-1309.

11. Amuta E, Houmsou R, Wama E, Ameh M (2014) Malarial Infection among Antenataland Maternity Clinics Attendees at the Federal Medical Centre, Makurdi, Benue State, Nigeria. Infect Dis Rep 6(1): 5050.

12. Oyefabi A, Sambo M, Sabitu K (2015) Effect of primary health care workers training on the knowledge and utilization of intermittent preventive therapy for malaria in pregnancy in Zaria, Nigeria. Journal of Medicine in the Tropics 17(1): 4-11.

13. Umaru M, Uyaiabasi GN (2015) Prevalence of Malaria in Patients Attending the General Hospital Makarfi, Makarfi Kaduna-State, North-Western Nigeria. American Journal of Infectious Diseases and Microbiology 3(1): 1-5.

14. Bates I, Bekoe V, Asamoa-Adu A (2004) Improving the accuracy of malaria-related laboratory tests in Ghana. Malar J 3: 38.

15. Cheesbrough M (2000) Hematological tests in District Laboratory practice in tropical countries (part 2). Cambridge University Press, pp: 267-347.

16. Tako EA, Zhou A, Lohoue J, Leke R, Taylor DW, et al. (2005) Risk factor for placental malaria and its effect on pregnancy outcome in Yaounde, Cameroon. Am J Trop Med Hyg 72(3): 236-242.

17. Iwuafor AA, Egwuatu CC, Nnachi AU, Lta Lo, Ogban GI, et al. (2016) Malaria Parasitaemia and the use of insecticide-treated nets (INTs) for malaria control amongst under-5 year old children in Calabar, Nigeria. BMC Infect Dis 16: 151.

18. Kovacs SD, Rijken MJ, Stergachis A (2010) Treating severe malaria in pregnancy. In: Okpere EE, Enabudoso EJ, Osemwenkha AP, (Eds.), Malaria in pregnancy, Nigerian Medical Journal 51(3): 109-113.

19. Chimere O, Oyibo WA, Anirlu RI, Agomo PU (2009) Prevalence of Malaria in Pregnant Women in Lagos, South-West Nigeria. Korean J Parasitol 47(2): 179-183.
20. Gajida AU, Iliyasu Z, Zoakah A (2010) Malaria among antenatal clients attending primary health care facilities in Kano state, Nigeria. Ann Afr Med 9(3): 188-193.

21. Iriemenam NC, Dosunmu AO, Oyibo WA, FagbenroBeyioku AF (2011) Knowledge, attitude, perception of malaria and evaluation of malaria parasitaemia among pregnant women attending antenatal care clinic in metropolitan Lagos, Nigeria. J Vector Borne Dis 48(1): 12-17.

22. Fana SA, Bunza MD, Anka SA, Imam AU, Nataala SU (2015) Prevalence and risk factors associated with malaria infection among pregnant women in a semiurban community of north-western Nigeria. Infect Dis Poverty 4: 24 .

23. Agomo CO, Oyibo WA (2013) Factors associated with risk of malaria infection among pregnant women in Lagos, Nigeria. Infect Dis Poverty-2 19: 1-8.

24. Alaku IA, Abdullahi AG, Kana HA (2015) Epidemiology of Malaria Parasites Infection among Pregnant Women in Some Part of Nasarawa State, Nigeria. Developing Country Studies 5(2): 30-33.

25. Aregawi M, Cibulskis RE, Otten M (2009) World Malaria Report 2009. World Health Organization.

26. Onyebuchi AK, Lawani LO, Iyoke CA, Onoh CR, Okeke NE (2014) Adherence to intermittent preventive treatment for malaria with sulphadoxine- pyrimethamine and outcome of pregnancy among parturients in South East Nigeria. Patient Preference and Adherence 8: 447-452.

27. Graves PM, Richards FO, Ngondi J, Emerson PM, Shargie EB, etal. (2009) Individual, household and environmental risk factors for malaria infection in Amhara, Oromia and SNNP regions of Ethiopia. Trans R Soc Trop Med Hyg 103(12): 1211-1220.

28. Osingada CP (2011) A comparison of determinant of malaria prevalence among pregnant women in two subcountties of Kumi District, Uganda. Makerere University, Kampala.

29. WHO (2004) A strategic framework for malaria prevention and control during pregnancy in the African region. World Health Organization, Regional Office for Africa, Brazzaville, pp: 27. 